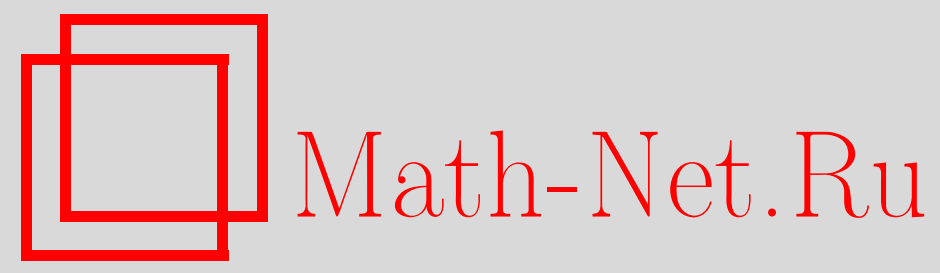

Г. М. Фельдман, Теорема Скитовича-Дармуа для компактных групп, Теория вероятн. и ее примен., 1996, том 41, выпуск 4, 901-906

DOI: https://doi.org/10.4213/tvp3244

Использование Общероссийского математического портала Math-Net.Ru подразумевает, что вы прочитали и согласны с пользовательским соглашением http://www . mathnet.ru/rus/agreement

Параметры загрузки:

IP: 3.85 .73 .92

26 апреля 2023 г., 13:51:57

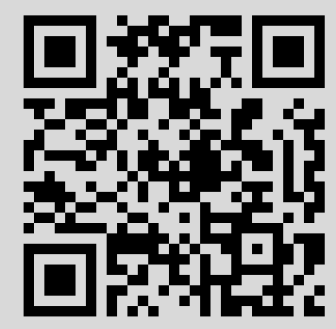


(c) $1996 \mathrm{r}$.

ФЕЛЬДМАН Г. М.*

\section{ТЕОРЕМА СКИТОВИЧА-ДАРМУА ПЛЯ КОМПАКТНЫХ ГРУПІІ ${ }^{1)}$}

Пусть $X$ - компактная абелева группа, $\xi_{j}$ - независимые случайные величины со значениями в $X, \alpha_{j}$ и $\beta_{j}$ - топологические автоморфизмы $X, L_{1}=$ $\alpha_{1}\left(\xi_{1}\right)+\cdots+\alpha_{s}\left(\xi_{s}\right), L_{2}=\beta_{1}\left(\xi_{1}\right)+\cdots+\beta_{s}\left(\xi_{s}\right)$. В работе дано полное описание групп $X$, на которых из независимости линейных форм $L_{1}$ и $L_{2}$ следует, что все $\xi_{j}$ имеют идемпотентные распределения. Эта теорема является групповым аналогом классических результатов Скитовича-Дармуа и Гурье-Олкина.

Ключевые слова и фразь: компактная абелева группа, случайная величина со значениями в группе, независимые линейные статистики.

Пусть $X$ - локально компактная сепарабельная абелева метрическая группа, $Y=X^{*}$ - ее группа характеров, $(x, y)$ - значение характера $y \in Y$ на элементе $x \in X$. Обозначим $\mathcal{M}^{1}(X)$ множество вероятностных распределений на группе $X$. Через $\widehat{\mu}(y)$ обозначим характеристическую функцию распределения $\mu \in \mathcal{M}^{1}(X)$. Обозначим через $\Gamma(X)$ множество гауссовских распределений на группе $X$, а через $I(X)$ - множество идемпотентных распределений на группе $X$, т.е. множество сдвигов распределений Хаара $m_{K}$ компактных подгрупп $K$ группы $X$.

ІІусть $\mathfrak{S}(X)$ - группа всех топологических автоморфизмов группы $X$. Предположим, что $\xi_{j}$ - независимые случайные величины со значениями в группе $X$ и с распределениями $\mu_{j}$. Рассмотрим линейные формы $L_{1}=\alpha_{1}\left(\xi_{1}\right)+\cdots+\alpha_{s}\left(\xi_{s}\right)$, $L_{2}=\beta_{1}\left(\xi_{1}\right)+\cdots+\beta_{s}\left(\xi_{s}\right)$, где $\alpha_{j}, \beta_{j} \in \mathfrak{S}(X)$, и сформулируем следующую задачу.

Дать полное описание групп $X$, которые обладают тем свойством, что из независимости линейных форм $L_{1}$ и $L_{2}$ следует, что все распределения $\mu_{j} \in I(X) * \Gamma(X)$. (Отметим, что если распределение $\mu \in I(X) * \Gamma(X)$, то $\mu$ инвариантно относительно некоторой компактной подгрупшы $K \subset X$, при естественном гомоморфизме $X \rightarrow X / K$ $\mu$ индуцирует на фактор-группе $X / K$ гауссовское распределение.)

Скитовичем и Дармуа было впервые обнаружено, что этим свойством обладает группа вещественных чисел $\mathbf{R}$. Затем аналогичный результат для групшы $\mathbf{R}^{n}, \boldsymbol{n}>\mathbf{1}$, был установлен Гурье и Олкиным (см. [1, гл. 3]). В [2] сформулированная задача была решена для конечных абелевых групп.

Целью настояшей работы является решение этой задачи в классе компактных абелевых групп.

Условимся о некоторых обозначениях. Если $H$ - замкнутая подгруппа группы $Y$, то обозначим через $A(X, H)=\{x \in X:(x, y)=1 \forall y \in H\}$ ее аннулятор. Через $C_{X}$ обозначим компоненту нуля группы $X$. Пусть $n-$ целое число. Обозначим $f_{n}: X \rightarrow X$ гомоморфизм, определяемый формулой $f_{n}(x)=n x$. Положим $X^{(n)}=f_{n}(X)$. Если $\alpha \in \mathfrak{S}(X)$, то сопряженный автоморфизм $\widetilde{\alpha} \in \mathfrak{S}(Y)$ определяется равенством $(x, \widetilde{\alpha}(y))=(\alpha(x), y)$ для любых $x \in X, y \in Y$. Отметим, что $\widetilde{\widetilde{\alpha}}=\alpha$. Подгруппа $G$ группы $X$; которая отображается в себя при всяком автоморфизме $\alpha \in \mathfrak{S}(X)$, называется характеристической подгруппой группы $X$. Через $I_{X}$ обозначим тождественный автоморфизм группы $X$. Через $Z(m)$ обозначим конечную циклическую группу из $m$ элементов $Z(m)=\{0,1, \ldots, m-1\}$ с операцией сложения по модулю $m$. Отметим, что $(Z(m))^{*} \approx Z(m)$.

*Физико-технический институт низких температур АН Украины, просп. Ленина, 47, 310164 Харьков, Украина.

1) Работа выполнена при частичной поддержке Международного научного фонда, грант U9S000. 
Пусть $p$ - простое число. Через $Z\left(p^{\infty}\right)$ обозначим рассматриваемую в дискретной топологии мультипликативную группу корней степени $p^{n}$ из единицы, где $n$ пробегает все неотрицательные целые числа. Нам понадобится также описание группы $p$-адических целых чисел $\Delta_{p}$. Группа $\Delta_{p}$ как множество совпадает с множеством последовательностей $x=\left(x_{0}, x_{1}, \ldots\right)$, где $x_{j} \in\{0,1, \ldots, p-1\}$, а сумма $z=x+y, x, y \in \Delta_{p}$, определяется следуюшим образом. Пусть $x_{0}+y_{0}=p t_{0}+z_{0}$, где $z_{0} \in\{0,1, \ldots, p-1\}, t_{0} \in Z$. Предположим, что числа $z_{0}, z_{1}, \ldots, z_{k}, t_{0}, t_{1}, \ldots, t_{k}$ уже определены. Тогда положим $x_{k+1}+y_{k+1}+t_{k}=p t_{k+1}+z_{k+1}$, где $z_{k+1} \in$ $\{0,1, \ldots, p-1\}, t_{k+1} \in Z$. Тем самым по индукции определена последовательность $z=\left(z_{0}, z_{1}, \ldots\right) \in \Delta_{p}$. Рассматриваемая в тихоновской топологии, группа $\Delta_{p}$ компактна, абелева и вполне несвязна. Группы $Z\left(p^{\infty}\right)$ и $\Delta_{p}$ являются группами характеров одиа другой:

$$
(x, y)=\exp \left\{\frac{2 \pi i k}{p^{n}}\left(y_{0}+y_{1} p+\cdots+y_{n-1} p^{n-1}\right)\right\}
$$

где $x=\exp \left\{2 \pi i k / p^{n}\right\} \in Z\left(p^{\infty}\right), y=\left(y_{0}, y_{1}, \ldots\right) \in \Delta_{p}$ (см. $\left.[3, \S 10]\right)$.

Пусть $\left\{X_{i}, i \in I\right\}-$ семейство групп. Обозначим через $P_{i \in I} X_{i}$ - прямое произведение групп $X_{i}$, а через $P_{i \in I}^{*} X_{i}$ - слабое прямое произведение групп $X_{i}$ (напомним, что $x=\left(x_{1}, \ldots, x_{i}, \ldots\right) \in P_{i \in I}^{*} X_{i}$, если $x_{i} \neq 0$ лишь для конечного числа индексов $i \in I$ ).

Периодическая группа $X$ называется $p$-примарной, если порядок любого ее элемента есть степень простого числа $p$. Группа $X$ называется делимой, если $X^{(n)}=X$ для любого $n=2,3, \ldots$ Группа $X$ называется редуцированной, если она не имеет ненулевых делимых подгрупп.

Обозначим через $E_{x}$ вырожденное распределение, сосредоточенное в точке $x \in$ $X$, а через $D(X)$ - множество вырожденных распределений на группе $X$. Через $\sigma(\mu)$ обозначим носитель распределения $\mu$, а через $\bar{\mu}$ - распределение, определяемое формулой $\bar{\mu}(E)=\mu(-E)$ для любого борелевского множества $E \subset X$. Отметим, что $\widehat{\bar{\mu}}(y)=\overline{\widehat{\mu}(y)}$. Заметим также, что если группа $X$ вполне несвязна, то $\Gamma(X)=D(X)[4]$.

Для удобства ссылок сформулируем в виде лемм следующие хорошо известные утверждения.

Лемма 1. Если $H-$ замкнутая подаруппа группы $Y, \mu \in \mathcal{M}^{1}(X)$ u $\widehat{\mu}(y) \equiv 1$ на $H$, mо $\sigma(\mu) \subset A(X, H)$.

Лемма 2. Пусть $G$ - борелевская подеруппа в $X$. Если $\mu=\mu_{1} * \mu_{2}, \mu \in \mathcal{M}^{1}(X)$ u распределение $\mu$ сосредоточено на $G$, то распределения $\mu_{j}$ можно так заменить их сдвигами $\mu_{j}^{\prime}=\mu_{j} * E_{x_{j}}$, что $\mu=\mu_{1}^{\prime} * \mu_{2}^{\prime}$, и распределения $\mu_{j}^{\prime}$ сосредоточены на $G$.

Отметим следующий факт. Так же как и в классическом случае, условие независимости линейных форм $L_{1}=\xi_{1}+\cdots+\xi_{\text {s }}$ и $L_{2}=\delta_{1}\left(\xi_{1}\right)+\cdots+\delta_{s}\left(\xi_{s}\right)$, где $\xi_{j}-$ независимые случайные величины со значениями в групте $X$ и с распределениями $\mu_{j}$, а $\delta_{j} \in \mathfrak{S}(X)$, равносильно тому, что характеристические функции $\widehat{\mu}_{j}(y)$ удовлетворяют уравнению

$$
\prod_{j=1}^{s} \widehat{\mu}_{j}\left(u+\tilde{\delta}_{j}(v)\right)=\prod_{j=1}^{s} \widehat{\mu}_{j}(u) \prod_{j=1}^{s} \widehat{\mu}_{j}(\tilde{\delta}(v)) ; \quad u, v \in Y,
$$

где $\widetilde{\delta}_{j}$ - сопряженные к $\delta_{j}$ автоморфизмы.

Условимся о следующем. Нас будут интересовать решения уравнения (1) на группе $Y$. Ясно, что при этом группу $Y$ можно заменить топологически изоморфной группой. Это позволяет, например, в ситуации, когда $X=\Delta_{p}$, а $Y \approx Z\left(p^{\infty}\right)$, считать, что $Y=Z\left(p^{\infty}\right)$, или, в ситуани, когда $X=F \times G$, а следовательно, и $Y \approx F^{*} \times G^{*}$, считать, что $Y=F^{*} \times G^{*}$, и т.п. Отметим, что если $X$ - компактная группа, то $Y$ - счетная дискретная групна.

Теорема 1. Пусть $\xi_{j}-$ независимые случаймые величины со зяачениями в компактной аруппе $X$ и с распределениями $\mu_{j}, L_{1}=\alpha_{1}\left(\xi_{1}\right)+\cdots+\alpha_{s}\left(\xi_{s}\right), L_{2}=$ 
$\beta_{1}\left(\xi_{1}\right)+\cdots+\beta_{s}\left(\xi_{s}\right)$, где $\alpha_{j}, \beta_{j} \in \mathfrak{S}(X)$. Предположим, ито линеймые формы $L_{1} u$ $L_{2}$ мезависимы. Тогда

(I) если группа $X$ топологически изоморфна одной из групп:

$$
\begin{array}{ll}
\text { (a) } & Z\left(2^{m_{1}}\right) \times \cdots \times Z\left(2^{m_{n}}\right), \quad 0 \leqslant m_{1}<\cdots<m_{n}, \\
\text { (b) } & \Delta_{2} \times Z\left(2^{m_{1}}\right) \times \cdots \times Z\left(2^{m_{n}}\right), \quad 0 \leqslant m_{1}<\cdots<m_{n},
\end{array}
$$

mо все $\mu_{j} \in D(X)$

(II) если гpynпа $X$ mопологически изоморфка аруппе $Z(3) \times G$, где $G$ - одна из арупп вида (a) или (b), то либо все $\mu_{j} \in D(X)$, либо распределения $\mu_{j}$ можно так заменить их сдвигами $\mu_{j}^{\prime}$, что $\mu_{j_{1}}^{\prime}=\mu_{j_{2}}^{\prime}=m_{Z(3)}$, по крайней мере, для двух распределений $\mu_{j_{1}}^{\prime}$ и $\mu_{j_{2}}^{\prime}$, а остальные $\mu_{j}^{l}$ прочзвольны, причем все носители $\sigma\left(\mu_{j}^{\prime}\right) \subset$ $Z(3), j=1,2, \ldots, s$.

Теорема 1 точна в следуюшем смысле.

Теорема 2. Пусть компактная группа $X$ топологически не изоморфна группам, перечисленмьм в теореме 1. Тогда существуют такие независимье случаймые величины $\xi_{j}$ со зяачениями в аруппе $X$ и с распределеяиями $\mu_{j}$ и автоморфизмы $\delta_{j} \in \mathfrak{S}(X)$, что линейные формы $L_{1}=\xi_{1}+\cdots+\xi_{s} u L_{2}=\delta_{1}\left(\xi_{1}\right)+\cdots+\delta_{s}\left(\xi_{s}\right)$ независимы, а все распределенив $\mu_{j} \notin I(X) * \Gamma(X)$.

Для доказательства теоремы 1 нам понадобится

Лемма 3 [2]. Пусть $X=Z(3), \xi_{j}$ - независимые случайные величины со значемиями в группе $X$ и с распределениями $\mu_{j}, \alpha_{j}, \beta_{j} \in \mathfrak{S}(X)$. Тогда из независимости лияеймых форм $L_{1}=\alpha_{1}\left(\xi_{1}\right)+\cdots+\alpha_{s}\left(\xi_{s}\right)$ и $L_{2}=\beta_{1}\left(\xi_{1}\right)+\cdots+\beta_{s}\left(\xi_{s}\right)$ вытекает, ито либо все $\mu_{j} \in D(X)$, либо $\mu_{j_{1}}=\mu_{j_{2}}=m_{X}$, по крайней мере, для двух распределений $\mu_{j_{1}}$ и $\mu_{j_{2}}$, а остальные распределения $\mu_{j}$ произвольны.

Д ок аз а т ел ь с т в о те о р е мы 1. Полагая $\eta_{j}=\alpha_{j}\left(\xi_{j}\right), j=1,2, \ldots, s$, мы сведем доказательство к случаю, когда линейные формы имеют вид $L_{1}=\xi_{1}+$ $\cdots+\xi_{s}$ и $L_{2}=\delta_{1}\left(\xi_{1}\right)+\cdots+\delta_{s}\left(\xi_{s}\right)$, где $\delta_{j} \in \mathfrak{S}(X)$.

Докажем (I). Положим $\nu_{j}=\mu_{j} * \bar{\mu}_{j}$. Тогда $\widehat{\nu}_{j}(y)=\left|\widehat{\mu}_{j}(y)\right|^{2} \geqslant 0$. Так как характеристические функци $\overline{\widehat{\mu}_{j}(y)}$ удовлетворяют уравнению (1), то уравнению (1) будут удовлетворять и характеристические функции $\widehat{\nu}_{j}(y)$. Это позволяет с самого начала считать, что все $\widehat{\mu}_{j}(y) \geqslant 0$. Теорема будет доказана, если мы установим, что при этом предположении $\mu_{j}=E_{0}, j=1,2, \ldots, s$. Утверждение (Іа) доказано в [2]. Докажем (Ib). Предположим вначале, что $X=\Delta_{2}$. Тогда $Y=Z\left(2^{\infty}\right)$ и имеют место вложения

$$
Z(2) \subset Z(4) \subset \cdots \subset Z\left(2^{n}\right) \subset \cdots \subset Z\left(2^{\infty}\right) .
$$

Очевидно, что $Z\left(2^{n}\right)$ является характеристической подгруппой групшы $Z\left(2^{\infty}\right)$ при любом натуральном $n$. Рассмотрим сужение уравнения (1) на подгруппу $Z\left(2^{n}\right)$ и воспользуемся (Та). Имеем $\widehat{\mu}_{j}(y) \equiv 1, y \in Z\left(2^{n}\right)$, а значит, $\widehat{\mu}_{j}(y) \equiv 1, y \in Z\left(2^{\infty}\right)$, т.е., $\mu_{j}=E_{0}, j=1,2, \ldots, s$.

Пусть

$$
X=\Delta_{2} \times Z\left(2^{m_{1}}\right) \times \cdots \times Z\left(2^{m_{n}}\right), \quad 0 \leqslant m_{1}<\cdots<m_{n}
$$

Тогда

$$
Y=Z\left(2^{\infty}\right) \times Z\left(2^{m_{1}}\right) \times \cdots \times Z\left(2^{m_{n}}\right)
$$

Поскольку $Z\left(2^{\infty}\right)$ - максимальная делимая подгруппа $Y$, то она является характеристической подтруппой. Рассмотрим сужение уравнения (1) на подгруппу $Z\left(2^{\infty}\right)$. Как отмечено выше, все $\widehat{\mu}_{j}(y) \equiv 1$ при $y \in Z\left(2^{\infty}\right)$. Значит, по лемме 1 ,

$$
\sigma\left(\mu_{j}\right) \subset A\left(X, Z\left(2^{\infty}\right)\right)=Z\left(2^{m_{1}}\right) \times \cdots \times Z\left(2^{m_{n}}\right) .
$$


Так как подгруппа $Z\left(2^{m_{1}}\right) \times \cdots \times Z\left(2^{m_{n}}\right)$, очевидно, является характеристической подгруппой группы $X$, то доказательство, таким образом, сводится к уже рассмотренному случаю (Ia).

Докажем (II). Как и при доказательстве (I), положим $\nu_{j}=\mu_{j} * \bar{\mu}_{j}$. Тогда $\widehat{\nu}_{j}(y) \geqslant$ 0 , и характеристические функци $\widehat{\nu}_{j}(y)$ также удовлетворяют уравнению (1).

Пусть для определенности $X=Z(3) \times \Delta_{2} \times Z\left(2^{m_{1}}\right) \times \cdots \times Z\left(2^{m_{n}}\right), 0 \leqslant m_{1}<\cdots<$ $m_{n}$. Тогда $Y=Z(3) \times Z\left(2^{\infty}\right) \times Z\left(2^{m_{1}}\right) \times \cdots \times Z\left(2^{m_{n}}\right)=Z(3) \times H$. Очевидно, что $H$ - характеристическая подгруппа группы $Y$. Рассматривая сужение уравнения (1) для характеристических функций $\widehat{\nu}_{j}(y)$ на подгруппу $H$ и учитывая, что в случае (I) теорема уже доказана, заключаем, что все $\hat{\nu}_{j}(y) \equiv 1$ при $y \in H$. По лемме 1 имеет место включение $\sigma\left(\nu_{j}\right) \subset A(X, H)=Z(3)$. Поскольку $Z(3)$ - характеристическая подгруппа группы $X$, то, по лемме 3 , либо все $\nu_{j}=E_{0}$, и тогда $\mu_{j} \in D(X), j=$ $1,2, \ldots, s, \pi и б о \nu_{j_{1}}=\nu_{j_{2}}=m_{Z(3)}$ по крайней мере для двух распределений $\nu_{j_{1}}$ и $\nu_{j_{2}}$, а остальные $\nu_{j}$ произвольны. Для завершения доказательства теорема остается сослагься на лемму 2.

Доказательство теоремы 2 опирается на следующие утверждения.

Предложение 1 [2]. Пусть $K$ - ненулевая компактная подерупna аруппь $X$, $K \not Z(3), L=K^{*}$. Предположим, что группа $\mathcal{S}(K)$ содержит такое конечное подмножество $A$, ито

1) любой топологический автоморфизм $\alpha \in A$ продолжается до топологического автоморфизма группь $X$;

2) для пюбого $l \in L, l \neq 0$, суиестьует такой топологический автоморфизм $\alpha \in A$, ито $\widetilde{\alpha}(l) \neq l$.

Тогда сушествуют такие пезависимые случайные величины $\xi_{j}$ со значениями в группе $X$ и с распределениями $\mu_{j}$ и топологические автоморфизмы $\delta_{j} \in \mathfrak{S}(X)$, что лияеймые формы $L_{1}=\xi_{1}+\cdots+\xi_{s} u L_{2}=\delta_{1}\left(\xi_{1}\right)+\cdots+\delta_{s}\left(\xi_{s}\right)$ независимы, а все распределекия $\mu_{j} \notin I(X) * \Gamma(X)$.

Лемма 4. Пусть группа $X$ содержит такую ненулевую компактную подерупnу $K$, что $K \not z Z(3)$ и $K^{(2)}=K$. Положим $A=\left\{-I_{K}\right\}$. Тогда $K$ и А удовлетворяют условиям 1) и 2) предложения 1 .

Д оказате льст в о. Условие 1), очевидно, выполнено. Пусть $L=K^{*}$. Так как $K^{(2)}=K$, то $\{l \in L: 2 l=0\}=\{0\}$, т.е. $-l \neq l$ для любого $l \in L, l \neq 0$. Следовательно, условие 2) также выполнено.

Д ок а зат т лі ь т в о т е о р е м ы 2. Если $C_{X} \neq\{0\}$, то $C_{X}$ - компактная подгруппа в группе $X, C_{X} \not \notin Z(3)$, и для любото натурального $n$ вылолнено $C_{X}^{(n)}=C_{X}$. Утверждение теоремы вытекает в этом случае из леммы 4 и предложения 1 .

Предположим поэтому, что $C_{X}=\{0\}$. Тогда $Y$ - счетная дискретная периодическая группа. Представим группу $Y$ в виде слабого прямого произведения своих p-примарных подтрупп $Y_{p}: Y=\mathrm{P}_{p \in \mathcal{P}}^{*} Y_{p}$, где $\mathcal{P}$ - множество всех простых чисел. Отсюда следует, что $X=\mathrm{P}_{p \in \mathcal{P}} X_{p}$, где $X_{p} \approx Y_{p}^{*}$. Отметим, что при любом $p \geqslant 3$ выполнено равенство $X_{p}^{(2)}=X_{p}$. Поэтому, если $X_{3} \neq\{0\}$ и $X_{3} \neq Z(3)$ или $X_{p} \neq\{0\}$ хотя бы при одном $p>3$, то утверждение теоремы вытекает из леммы 4 и предложения 1. Следовательно, в доказательстве нуждается лишь случай, когда $X=X_{2} \times X_{3}$, где либо $X_{3}=\{0\}$, либо $X_{3}=Z(3)$.

Рассмотрим группу $Y_{2}$. Представим ее в виде прямого произведения $Y_{2}=D \times N$, где $D$ - максимальная делимая подгруппа группы $Y_{2}$, а $N$ - редуцированная 2примарная группа, Отметим, что группа $D$ изоморфна слабому прямому произведению групп $Z\left(2^{\infty}\right)$, а группа $N$ счетна.

Если группа $N$ ограничена, т.е. порядки всех элементов группы $N$ ограничены в совокупности, то по теореме Прюфера $[5$, с. 107] группа $N$ является слабым прямым произведением циклических групщ. Если же группа $N$ неограничена, то группа $N$ 
имеет прямой сомножитель, являюшийся неограниченным слабым прямым произведением циклических групп $[6$, с. 83$]$.

Поскольку группа $X$ топологически не изоморфна группам, перечисленным в теореме 1 , то из сказанного выше следует, что группа $X$ содержит в качестве топологического прямого сомножителя подгруппу $K$, топологически изоморфную одной из групा

(a) $\quad \Delta_{2} \times \Delta_{2}$

(c) $\quad Z\left(2^{k}\right) \times Z\left(2^{k}\right)$.

Так как $K$ - топологический прямой сомножитель в $X$, то любой топологический автоморфизм группы $K$ продолжается до топологического автоморфизма группы $X$. Поэтому условие 1) предложения 1 выполнено для любого подмножества $A \subset \mathfrak{S}(K)$.

Проверим, что в каждом из случаев (a)-(c) можно построить такое конечное подмножество $B \subset \mathfrak{S}(L), L=K^{*}$, что для любого $l \in L, l \neq 0$, существует автоморфизм $b \in B$, для которого $b(l) \neq l$. Тем самым будет доказано, что условие 2$)$ предложения 1 выполнено для $A=\{a \in \mathfrak{S}(K): a=\widetilde{b}, b \in B\}$.

(a) Имеем

$$
L=Z\left(2^{\infty}\right) \times Z\left(2^{\infty}\right) .
$$

Элементы группы $L$ будем обозначать через $l=\left(l_{1}, l_{2}\right) ; l_{i} \in Z\left(2^{\infty}\right)$. Рассмотрим автоморфизмы $b_{1}, b_{2} \in \mathfrak{S}(L)$, определяемые формулами:

$$
b_{1}\left(l_{1}, l_{2}\right)=\left(l_{2}, l_{1}\right), \quad b_{2}\left(l_{1}, l_{2}\right)=\left(l_{1}, l_{1}+l_{2}\right), \quad l=\left(l_{1}, l_{2}\right) \in L .
$$

Tогда, как легко видеть, можно положить $B=\left\{b_{1}, b_{2}\right\}$.

(b) Имеем

$$
L=\stackrel{\stackrel{P}{P}}{*}_{i=1}^{*} Z\left(2^{m_{i}}\right), \quad m_{i}<m_{i+1}, \quad i=1,2, \ldots .
$$

Элементы группы $L$ будем обозначать $l=\left(l_{1}, \ldots, l_{i}, \ldots\right), l_{i} \in Z\left(2^{m_{i}}\right)$. Рассмотрим автоморфизмы $b_{1}, b_{2} \in \mathfrak{S}(L)$, определяемые формулами:

$$
\begin{aligned}
b_{1}\left(l_{1}, \ldots, l_{i}, \ldots\right) & =\left(h_{1}, \ldots, h_{i}, \ldots\right), \quad \text { где } h_{2 i-1}=l_{2 i-1}, \\
h_{2 i} & =\left(l_{2 i-1} 2^{m_{2 i}-m_{2 i-1}}+l_{2 i}\right)\left(\bmod 2^{m_{2 i}}\right), \quad i=1,2, \ldots, \\
b_{2}\left(l_{1}, \ldots, l_{i}, \ldots\right) & =\left(h_{1}, \ldots, h_{i}, \ldots\right), \quad \text { где } \quad h_{1}=l_{1}, \quad h_{2 i}=l_{2 i} \\
h_{2 i+1} & =\left(l_{2 i} 2^{m_{2 i+1}-m_{2 i}}+l_{2 i+1}\right)\left(\bmod 2^{m_{2 i+1}}\right), \quad i=1,2, \ldots .
\end{aligned}
$$

По построению $h=\left(h_{1}, \ldots, h_{i}, \ldots\right) \in L$. Проверим, что у автоморфизмов $b_{1}$ и $b_{2}$ нет отличных от нуля общих неподвижных точек. Действительно, пусть $b_{1}(l)=l$. Для нахождения $l_{i}$ имеем систему

$$
\left\{\begin{array}{l}
l_{1}=l_{1} \\
l_{1} 2^{m_{2}-m_{1}}+l_{2} \equiv l_{2}\left(\bmod 2^{m_{2}}\right) \\
l_{3}=l_{3} \\
\ldots \ldots \ldots \ldots
\end{array}\right.
$$

Отсюда следует, что $l=\left(0, l_{2}, 0, l_{4}, 0, \ldots\right)$, где $l_{2 i}$ - произвольный элемент группы $Z\left(2^{m_{2 i}}\right)$. Аналогично получаем, что если $b_{2}(l)=l$, то $l=\left(l_{1}, 0, l_{3}, 0, \ldots\right)$, где $l_{2 i-1}-$ произвольный элемент группы $Z\left(2^{m_{2 i-1}}\right)$. Ясно, что если $b_{1}(l)=b_{2}(l)=l$, то $l=0$. Множество $B=\left\{b_{1}, b_{2}\right\}$ - искомое. 
(c) Имеем $L=Z\left(2^{k}\right) \times Z\left(2^{k}\right)$. Положим $B=\left\{b_{1}, b_{2}\right\}$, где автоморфизмы $b_{1}$ и $b_{2}$ строятся так же, как и в случае (а).

Утверждение теоремы вытекает теперь из предложения 1.

\title{
ФПИСОК ЛИТЕРАТУРЫ
}

1. Кагая А. М., Лияник Ю. В., Рао С. Р. Характеризационные задачи математической статистики. М.: Наука, 1972, 656 с.

2. Фельдман Г. М. К теореме Скитовича-Дармуа на абелевых группах. - Теория вероятн. и ее примен., 1992, т. 37 , в. 4, с. 695-708.

3. Хьюит Э., Росс К. Абстрактный гармонический анализ. Т.1. М.: Наука, 1975, $656 \mathrm{c}$.

4. Партасарати К. Р., Ранаа Рао Р., Варадхан С. Р.С . Распределения вероятностей на локально компактных абелевых группах. - Математика. Сб. переводов, 1965 , т. 9, в. 2, с. 115-146.

5. Фукс Л. Бесконечные абелевы группы. Т. 1. М.: Мир, 1974, 336 с.

6. Фукс Л. Бесконечные абелевы группы. Т. 2. М.: Мир, 1977, 416 с.

Поступила в редакцию 28.II.1995

(C) 1996 г.

ФилИМОНОВ Е. В.*

\section{ЭКСПОНЕНЩИАЛЬНАЯ ОЦЕНКА ДЛЯ РЕШЕНИЯ СТОХАСТИЧЕСКОГО ДИФФЕРЕНЦИАЛЬНОГО УРАВНЕНИЯ СО СКАЧКАМИ}

\begin{abstract}
Получены неасимптотические экспоненциальные оценки сверху для «хвоста» Функции распределения сильного решения стохастического дифференциального уравнения Ито с разрывным членом в виде интеграла по локальной мартингальной мере. Отдельно рассматриваются случаи ограниченных по совокупности переменных и неограниченно растуших коэффициентов исследуемого уравнения.
\end{abstract}

Клюиевые слова и фразы: стохастическое дифференциальное уравнение, не-

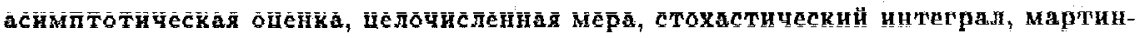
гал, формула Ито, случайный оператор, банахово пространство, неподвижная точкa.

Изучение свойств сильных решений стохастических дифференциальных уравнений Ито (СДУ) представляет существенный интерес как с теоретической, так и с практической точек зрения. Соответствующие результаты могут найти широкое применение в статистике случайных процессов, в теории надежности, в теории автоматического управления и ряде других приложений.

В [7] автором рассматривался класс СДУ типа $d \xi_{t}=a\left(t, \xi_{t}\right) d t+b\left(t, \xi_{t}\right) d w_{t}$, для которого были получены верхние неасимптотические ощенки «хвоста» функции распределения сильного решения. В настоящей работе подобные оценки выводятся для

*Обнинский институт атомной энергетики, Студенческий городок, 1, 249020 Обнинск, Калужская обл., Россия. 